Peter D. Cole, Veena Vijayanathan, Albert Einstein College of Medicine, Bronx, NY; Yaron Finkelstein, Hospital for Sick Children, University of Toronto Toronto; Philippe Robaey, Children's Hospital of Eastern Ontario, Ottawa, Ontario, Canada; Kristen E. Stevenson, Traci M. Blonquist, Donna S. Neuberg, Dana-Farber Cancer Institute (DFCl); Lewis B. Silverman, Stephen E. Sallan, DFCl, Boston Children's Hospital, Harvard Medical School; and Deborah P. Waber, DFCl, Harvard Medical School, Boston, MA.

Published online ahead of print at www.jco.org on May 18, 2015.

Support information appears at the end of this article.

Authors' disclosures of potential conflicts of interest are found in the article online at www.jco.org. Author contributions are found at the end of this article.

Corresponding author: Peter D. Cole, $M D$, Albert Einstein College of Medicine, 1300 Morris Park Ave, Bronx, NY 10461; e-mail: Peter.Cole@Einstein.YU .edu.

(C) 2015 by American Society of Clinical Oncology

0732-183X/15/3319w-2205w/\$20.00

DOI: $10.1200 / J C O .2014 .59 .0273$

\title{
Polymorphisms in Genes Related to Oxidative Stress Are Associated With Inferior Cognitive Function After Therapy for Childhood Acute Lymphoblastic Leukemia
}

Peter D. Cole, Yaron Finkelstein, Kristen E. Stevenson, Traci M. Blonquist, Veena Vijayanathan, Lewis B. Silverman, Donna S. Neuberg, Stephen E. Sallan, Philippe Robaey, and Deborah P. Waber

$$
\begin{array}{llllllll}
\text { A } & \text { B } & \text { S } & \text { T } & \text { R } & \text { A } & \text { C } & \text { T }
\end{array}
$$

\section{Purpose}

Survivors of childhood acute lymphoblastic leukemia (ALL) exhibit increased rates of neurocognitive deficits. This study was conducted to test whether interpatient variability in neurocognitive outcomes can be explained by polymorphisms in candidate genes conferring susceptibility to neurocognitive decline.

\section{Methods}

Neurocognitive testing was conducted in 350 pediatric leukemia survivors, treated on Dana-Farber Cancer Institute ALL Consortium Protocols 95-01 or 00-01. Genomic DNA was isolated from bone marrow collected at remission. Candidate polymorphisms were selected on the basis of prior literature, targeting genes related to drug metabolism, oxidative damage, altered neurotransmission, neuroinflammation, and folate physiology. Single nucleotide polymorphisms were detected using either a customized multiplexed Sequenom MassARRAY assay or polymerase chain reaction-based allelic discrimination assays. Multivariable logistic regression models were used to estimate the effects of genotype on neurocognitive outcomes, adjusted for the effects of demographic and treatment variables. False-discovery rate correction was made for multiple hypothesis testing, indicated as a $\mathrm{O}$ value.

\section{Results}

Inferior cognitive or behavioral outcomes were associated with polymorphisms in three genes related to oxidative stress and/or neuroinflammation: $N O S 3(\mathrm{IQ}, Q=0.008$; Vocabulary $Q=0.011$; Matrix Reasoning $Q=0.008$ ), SLCO2A1 (IQ $Q=0.043$; Digit Span $Q=0.006$; Block Design $Q=$ 0.076), and COMT (Behavioral Assessment System for Children-2 Attention $Q=0.080$; and Hyperactivity $Q=0.084$ ). Survivors homozygous for NOS3 894T, with at least one SLCO2A1 variant G allele or with at least one GSTP1 variant allele, had lower mean estimated IQ scores than those without these genotypes.

\section{Conclusion}

These data are consistent with the hypothesis that oxidative damage contributes to chemotherapy-associated neurocognitive decline among children with leukemia.

\section{J Clin Oncol 33:2205-2211. (C) 2015 by American Society of Clinical Oncology}

\section{INTRODUCTION}

Although the majority of children with acute lymphoblastic leukemia (ALL) can be cured, ${ }^{1}$ treatment-related toxicity remains a persistent challenge. Outcome studies document increased rates of deficits in attention, working memory, and processing complex novel information among childhood leukemia survivors, ${ }^{2-11}$ with the potential for lifelong impact on academic performance, occupational outcomes, and quality of life. There exists considerable interpatient variability in neurocognitive outcomes, some of which has been related to demographic factors (eg, female sex and young age $^{4,12}$ ) or treatment variables (eg, cranial irradiation therapy, ${ }^{4,13,14}$ high-dose intravenous methotrexate $\left.^{2,15}\right)$. These factors do not, however, reliably identify all childhood ALL survivors with neurocognitive deficits.

Over the past two decades, investigators have begun to explore genetic influences that may account for individual variation in neurocognitive outcomes. ${ }^{16}$ Krajinovic et $\mathrm{al}^{17}$ reported that the NOS3 894TT genotype was associated with significant change in IQ scores after completion of childhood ALL treatment, particularly among those 
patients who had been treated with cranial radiation. More recently, Krull et $\mathrm{al}^{18}$ identified associations among laboratory and questionnaire measures of attention and genetic polymorphisms in methionine synthase, monoamine oxidase $\mathrm{A}$, apolipoprotein $\mathrm{E}$, and glutathione $S$-transferases GSTT1 and GSTP1.

The aim of the this study was to expand our understanding of associations between prevalent genetic polymorphisms that could plausibly influence treatment-related toxicity and neurocognitive outcomes measured in a large cohort of childhood ALL survivors. Our selection of genetic variants was guided by an understanding of pathophysiologic mechanisms, ${ }^{19-22}$ focused on those likely to influence neurologic function. We thus selected prevalent variants within genes related to the two agents most frequently implicated in neurotoxicity of therapy for childhood ALL: methotrexate ${ }^{2,23}$ and corticosteroids. ${ }^{12}$ Methotrexate, by inhibiting methylation reactions dependent on folic acid, leads to an increase in the toxic amino acid homocysteine in blood and cerebrospinal fluid. ${ }^{24,25}$ Homocysteine may contribute to neurotoxicity through induction of oxidative damage to neuronal tissue and vascular endothelium, ${ }^{26,27}$ and through further metabolism to excitotoxic glutamate analogs. ${ }^{28,29}$ Steroids directly influence mood and memory through glucocorticoid receptors in the frontal cortex and hippocampus. ${ }^{12,30}$ Consequently, we probed polymorphisms in genes relevant to folate physiology, glucocorticoid pharmacodynamics, defense against oxidative stress, ${ }^{27,31}$ neuroinflammation, ${ }^{32}$ and neurotransmission. ${ }^{21}$ This panel of targeted variants also included genes that have previously been linked to $\mathrm{IQ}^{17}$ or attention ${ }^{18}$ among patients with childhood leukemia, as well as others that have not been previously investigated in this context.

\section{METHODS}

\section{Patients}

Children and adolescents between the ages of 1 and 18 years with newly diagnosed ALL were eligible for enrollment onto Dana-Farber Cancer Institute (DFCI) ALL Consortium Protocols $95-01^{33}$ and $00-01 .^{34}$ The protocols were approved by the institutional review boards of each participating institution, and informed consent was obtained from parents or guardians before study enrollment and the initiation of therapy. Patient characteristics, details of therapy, and disease outcomes have been described for both protocols. ${ }^{33,34}$ A total of 983 evaluable patients were enrolled. After completion of therapy, 381 patients ( 170 treated on $00-01^{35}$ and 211 on $95-01^{36}$ ) had neurocognitive testing, and 350 of these also had genomic DNA available for analysis. Patient characteristics are shown in Table 1, and a comparison with the remaining patients treated on 00-01 or 95-01 is shown in Appendix Table A1 (online only).

\section{Neurocognitive Assessments}

The neuropsychological testing protocols and outcomes have been described previously. ${ }^{35,36}$ Briefly, testing was initiated approximately 5 years after diagnosis. Patients with pre-existing neurodevelopmental problems that could impair cognitive function were excluded, as were patients who did not remain in remission. Informed consent for the neurocognitive assessment was obtained from each participant or each participant's guardian, and assent was obtained from minors.

We used a relatively brief neuropsychological battery to enhance reliability and comparability of data across institutions and to encourage compliance. Testing was conducted in Spanish or French for native speakers of those languages, using published instruments and language-specific norms. IQ was estimated on the basis of two subtests from the Wechsler scales. For the 95-01 protocol, IQ was estimated from the Vocabulary and Block Design subtests of the Wechsler Intelligence Scale for Children-III or Wechsler Adult Intelligence Scale. ${ }^{37}$ For the $00-01$ protocol, IQ was estimated from the Vocabulary and

\begin{tabular}{|lc|}
\hline \multicolumn{2}{|c|}{ Table 1. Patient Characteristics } \\
\hline \multicolumn{1}{|c}{ Characteristic } \\
\hline Total & No. (\%) \\
\hline DFCI ALL consortium treatment protocol & 350 \\
95-01 & $209(60)$ \\
00-01 & $141(40)$ \\
Age at initial diagnosis, years; median (range) & $4.2(1-17.99)$ \\
$<5$ & $210(60)$ \\
5-9.99 & $93(27)$ \\
10-17.99 & $47(13)$ \\
Sex & $159(45)$ \\
Female & $191(55)$ \\
Male & \\
Risk group & $225(64)$ \\
Standard risk & $125(36)$ \\
High risk & \\
Race/ethnicity & $308(88)$ \\
White & $8(2)$ \\
Black or African American & $6(2)$ \\
Asian & $28(8)$ \\
Other/unknown & $25(7)$ \\
Hispanic & \\
CNS prophylaxis & $176(50)$ \\
Intrathecal chemotherapy & $156(45)$ \\
Cranial radiation (12 or 18 Gy) & $18(5)$ \\
Not recorded/not given & \\
Corticosteroids & $277(79)$ \\
Prednisone & $73(21)$ \\
Dexamethasone & $0(0)$ \\
None & $10(6-25)$ \\
Age at time of neurocognitive testing, years; median (range) & \\
Years from diagnosis to testing, median (range) & $10(3)$ \\
Mother's education & \\
High school graduate/GED or less & \\
Some college or more & \\
Unknown & \\
\hline NOTE. Demographic and treatment variables are shown for patients included \\
in the genetic analyses. \\
Abbreviations: ALL, acute lymphoblastic leukemia; DFCl, Dana-Farber Cancer \\
Institute; GED, general equivalency diploma. \\
\hline
\end{tabular}

Matrix Reasoning subtests from the Wechsler Abbreviated Scale of Intelligence. We also included the age-appropriate Wechsler Digit Span, ${ }^{38}$ a measure of working memory, and the Rey-Osterrieth Complex Figure, ${ }^{39}$ a measure of the ability to integrate complex material. To assess psychosocial adjustment in everyday life, we administered the Behavioral Assessment System for Children-2 (BASC-2), ${ }^{40}$ a structured parent questionnaire that yields scales characterizing mood and behavior symptoms as well as positive adaptation. The BASC-2 scales that assess attention symptoms, Attention and Hyperactivity, were included in the present analysis because of prior reports of genetic associations with attention problems. Parents were asked whether the child was currently receiving special education services.

\section{Genomic DNA}

Genomic DNA was isolated from bone marrow collected at the end of induction for the purpose of detecting minimal residual disease. All samples were deidentified and coded before shipment for this study. Consequently, the investigators conducting the genetic analyses (P.D.C., V.V.) remained blinded to demographic information and neurocognitive outcomes.

\section{Candidate Gene Selection and Target Genotypes}

Candidate genetic variants were selected through a nonexhaustive literature review, with the following criteria: (1) variants present in genes related to 


\begin{tabular}{|c|c|c|c|c|c|c|c|c|c|c|}
\hline \multirow[b]{2}{*}{ Gene } & \multirow[b]{2}{*}{ refSNP } & \multirow[b]{2}{*}{ Method } & \multirow{2}{*}{$\begin{array}{l}\text { Total Calls, } \\
\text { No. }(\%)\end{array}$} & \multirow{2}{*}{$\begin{array}{c}\text { Target Versus Nontarget } \\
\text { Genotype }\end{array}$} & \multicolumn{2}{|c|}{ Wild Type } & \multicolumn{2}{|c|}{ Heterozygous } & \multicolumn{2}{|c|}{ Homozygous } \\
\hline & & & & & & No. $(\%)$ & & No. $(\%)$ & & No. $(\%)$ \\
\hline \multicolumn{11}{|c|}{ Genes Related to Folate Metabolism } \\
\hline MTHFR & 1801131 & iPLEX & $295(84 \%)$ & $\mathrm{AC} / \mathrm{CC} \vee \mathrm{AA}$ & AA & $152(51 \%)$ & $A C$ & $117(40 \%)$ & $\mathrm{CC}$ & $26(9 \%)$ \\
\hline MTHFR & 1801133 & TaqMan & $274(78 \%)$ & $\mathrm{TT} \vee \mathrm{CC} / \mathrm{CT}$ & $\mathrm{CC}$ & $100(36 \%)$ & CT & $142(52 \%)$ & $\mathrm{TT}$ & $32(12 \%)$ \\
\hline SLC19A1 & 1051266 & iPLEX & $283(81 \%)$ & $A A \vee A G / G G$ & GG & $81(29 \%)$ & AG & $131(46 \%)$ & AA & $71(25 \%)$ \\
\hline TCN2 & 1801198 & iPLEX & $307(88 \%)$ & $\mathrm{GG} \vee \mathrm{CC} / \mathrm{CG}$ & $\mathrm{CC}$ & $113(37 \%)$ & CG & $136(44 \%)$ & GG & $58(19 \%)$ \\
\hline TS & 28bp repeat & PCR & $207(69 \%)^{*}$ & $3 R / 3 R \vee 2 R / 2 R$ or $2 R / 3 R$ & $2 \mathrm{R} / 2 \mathrm{R}$ & $36(17 \%)$ & $2 \mathrm{R} / 3 \mathrm{R}$ & $133(64 \%)$ & $3 R / 3 R$ & $38(18 \%)$ \\
\hline \multicolumn{11}{|c|}{ Genes Related to Glucocorticoids } \\
\hline$A B C B 1$ & 1045642 & iPLEX & $301(86 \%)$ & $\mathrm{TT} \vee \mathrm{CC} / \mathrm{CT}$ & $\mathrm{CC}$ & $91(30 \%)$ & CT & $140(47 \%)$ & $\mathrm{TT}$ & $70(23 \%)$ \\
\hline NR3C1 & 41423247 & iPLEX & $296(85 \%)$ & $\mathrm{CG} / \mathrm{GG} \vee \mathrm{CC}$ & $\mathrm{CC}$ & $123(42 \%)$ & CG & $140(47 \%)$ & GG & $33(11 \%)$ \\
\hline \multicolumn{11}{|c|}{ Genes Related to Neurotransmission } \\
\hline COMT & 4680 & TaqMan & $280(80 \%)$ & $A A \vee A G / G G$ & GG & $76(27 \%)$ & AG & $161(58 \%)$ & AA & $43(15 \%)$ \\
\hline GRIN2B & 10193895 & iPLEX & $302(86 \%)$ & $A G / G G \vee A A$ & AA & $279(92 \%)$ & AG & $22(7 \%)$ & GG & $1(1 \%)$ \\
\hline MAOA & 1137070 & TaqMan & $267(76 \%)$ & $\mathrm{CT} / \mathrm{TT} \vee \mathrm{CC}$ & $\mathrm{CC}$ & $159(60 \%)$ & CT & $55(21 \%)$ & $\mathrm{TT}$ & $53(20 \%)$ \\
\hline SLC6A4 & 1042173 & TaqMan & $243(69 \%)$ & $\mathrm{AA} \vee \mathrm{CA} / \mathrm{CC}$ & $\mathrm{CC}$ & $43(18 \%)$ & CA & $135(56 \%)$ & AA & $65(27 \%)$ \\
\hline \multicolumn{11}{|c|}{ Genes Related to Inflammation or Oxidative Damage } \\
\hline APOE & 7412 & TaqMan & $281(80 \%)$ & $\mathrm{CT} / \mathrm{TT} \vee \mathrm{CC}$ & $\mathrm{CC}$ & $242(86 \%)$ & СТ & $39(14 \%)$ & $\mathrm{TT}$ & $0(0 \%)$ \\
\hline$A P O E$ & 429358 & TaqMan & $325(93 \%)$ & $\mathrm{CC} / \mathrm{CT} \vee \mathrm{TT}$ & $\mathrm{TT}$ & $249(77 \%)$ & TC & $73(22 \%)$ & $\mathrm{CC}$ & $3(1 \%)$ \\
\hline GSTP1 & 1138272 & TaqMan & $280(80 \%)$ & $\mathrm{CT} / \mathrm{TT} \vee \mathrm{CC}$ & $\mathrm{CC}$ & $220(79 \%)$ & CT & $48(17 \%)$ & $\mathrm{TT}$ & $12(4 \%)$ \\
\hline GSTP1 & 1695 & TaqMan & $251(72 \%)$ & $A G / G G \vee A A$ & AA & 121 (48\%) & $A G$ & $111(44 \%)$ & GG & 19 (8\%) \\
\hline HFE & 1799945 & iPLEX & $304(87 \%)$ & $\mathrm{CG} / \mathrm{GG} \vee \mathrm{CC}$ & $\mathrm{CC}$ & $235(77 \%)$ & CG & $63(21 \%)$ & GG & $6(2 \%)$ \\
\hline HFE & 1800562 & iPLEX & $302(86 \%)$ & $\mathrm{AA} / \mathrm{AG} \vee \mathrm{GG}$ & GG & 269 (89\%) & $A G$ & 33 (11\%) & AA & $0(0 \%)$ \\
\hline ILIB & 1143627 & TaqMan & $269(77 \%)$ & $\mathrm{GG} \vee \mathrm{AA} / \mathrm{AG}$ & AA & $104(39 \%)$ & $A G$ & $73(27 \%)$ & GG & $92(34 \%)$ \\
\hline$I L 1 B$ & 1143634 & TaqMan & 327 (93\%) & $A A \vee A G / G G$ & GG & 175 (54\%) & $A G$ & $100(31 \%)$ & AA & $52(16 \%)$ \\
\hline ILIRN & 380092 & TaqMan & $291(83 \%)$ & TT $v$ AA/AT & AA & $131(45 \%)$ & AT & $123(42 \%)$ & $\mathrm{TT}$ & $37(13 \%)$ \\
\hline NOS3 & 1799983 & iPLEX & $300(86 \%)$ & $\mathrm{TT} v \mathrm{GG} / \mathrm{GT}$ & GG & 137 (46\%) & GT & $133(44 \%)$ & TT & $30(10 \%)$ \\
\hline NOO1 & 1800566 & TaqMan & 312 (89\%) & $\mathrm{CT} / \mathrm{TT} \vee \mathrm{CC}$ & $\mathrm{CC}$ & $189(61 \%)$ & $\mathrm{CT}$ & $115(37 \%)$ & $\mathrm{TT}$ & $8(3 \%)$ \\
\hline$S L C O 2 A 1$ & 7625035 & iPLEX & $300(86 \%)$ & $A G / G G \vee A A$ & AA & $182(61 \%)$ & $A G$ & $93(31 \%)$ & GG & $25(8 \%)$ \\
\hline
\end{tabular}

pathways presumed to be relevant to treatment-induced cognitive deficits, including antifolate or corticosteroid pharmacodynamics, oxidative stress, neurotransmission, or neuroinflammation; (2) variants known to be associated with altered function of the gene product; and (3) variants with a population prevalence of at least $10 \%$. Twenty-eight genetic variants were selected for analysis (Table 2). For each polymorphism, an a priori hypothesis was established regarding a target genotype (ie, whether risk of neurocognitive deficits would be associated with being homozygous for the variant allele or with having $\geq$ one copy of the variant allele).

\section{Genotyping}

Thirteen single-nucleotide polymorphisms (SNPs) were detected using Sequenom MassARRAY iPLEX Platform (Sequenom Bioscience, San Diego, CA), a customized multiplexed assay. Briefly, initial multiplexed polymerase chain reaction (PCR) was performed in 5 - $\mu \mathrm{L}$ reactions on 384 -well plates containing 5 ng of genomic DNA, 0.5 U HotStart Taq polymerase (Qiagen, Valencia, CA), $100 \mathrm{nmol} / \mathrm{L}$ primers, $1.25 \times$ HotStart Taq buffer, $1.625 \mathrm{mmol} / \mathrm{L}$ $\mathrm{MgCl} 2$, and $500 \mu \mathrm{mol} / \mathrm{L}$ deoxyribonucleotide triphosphates. After enzyme activation at $94^{\circ} \mathrm{C}$ for 15 minutes, DNA was amplified with 45 cycles of $94^{\circ} \mathrm{C} \times$ 20 seconds, $56^{\circ} \mathrm{C} \times 30$ seconds, $72^{\circ} \mathrm{C} \times 1$ minute, followed by a 3-minute extension at $72^{\circ} \mathrm{C}$. Single-base extension (SBE) was carried out in $9-\mu \mathrm{L}$ reactions, by addition of SBE primers, iPLEX enzyme and buffers (Sequenom). SBE products were then assayed using the MassARRAY Compact system, and mass spectra were analyzed using TYPER software (Sequenom), to generate genotype calls and allele frequencies. An additional 14 SNPs were detected using PCR-based allelic discrimination assays (Life Technologies, Grand Island, NY), following the manufacturer's instructions. Genotype calls and allele frequencies were analyzed with Applied Biosystems TaqMan Genotyper Software v1.3 (Life Technologies). SNP genotypes with questionable or lowprobability calls (ie, where a specific genotype could not be assigned) were repeated with additional DNA, or excluded from further analysis.

The number of 28-base pair (bp) nucleotide repeats within the $5^{\prime}$ untranslated region of the gene for thymidylate synthase was assessed by PCR-product length analysis. PCR primer sequences were 5'-GTGGCTCCTGCGTTTCCCCC-3' ${ }^{\prime}$-GCTCCGAGCCGGCCACAGGCATGGCGCGG-3' . The 20- $\mu \mathrm{L}$ PCR reaction mixture contained $12.5 \mu \mathrm{L}$ of master mix, $1.5 \mu \mathrm{L}$ of the primer mix, and 2 ng of genomic DNA. Each of the 35 PCR cycles consisted of 1 minute at $96^{\circ} \mathrm{C}, 30$ seconds at $60^{\circ} \mathrm{C}$ and 1 minute at $72^{\circ} \mathrm{C}$. After the reaction, the mixture was incubated at $72^{\circ} \mathrm{C}$ for 5 minutes. The amplified DNA fragments were analyzed by electrophoresis on a $4 \%$ agarose gel. The sizes of the amplified fragments ( 250 or $220 \mathrm{bp}$ for the $3 \mathrm{R}$ and $2 \mathrm{R}$ products, respectively) were determined by reference to molecular weight markers.

\section{Statistical Analyses}

The neurocognitive outcome variables were dichotomized for purposes of analysis. Impaired performance on the Rey-Osterrieth Complex Figure test was defined by scores less than or equal to those for the 25 th percentile for age. For all other tests, impaired function was defined by scores more than one 


\begin{tabular}{|c|c|c|c|c|c|c|}
\hline Measure & No. & Median (range) & Percentile (25th, 75th) & Mean (SD) & Impairment, No. $(\% ; 95 \% \mathrm{Cl})$ & $P$ \\
\hline 10 & 344 & $103.0(59-152)$ & $94.0,113.0$ & $103.3(15.1)$ & $39(11 ; 8$ to 15$)$ & .99 \\
\hline Wechsler Digit Span & 349 & $9.0(1-18)$ & $7.0,11.0$ & $9.2(3.0)$ & $103(30 ; 25$ to 35$)$ & $<.0001$ \\
\hline Wechsler Vocabulary & 347 & $11.0(2-19)$ & $9.0,13.0$ & $10.7(3.1)$ & $51(15 ; 11$ to 19$)$ & .79 \\
\hline Wechsler Block Design & 209 & $10.0(2-19)$ & $8.0,12.0$ & $10.3(3.2)$ & $36(17 ; 13$ to 23$)$ & .34 \\
\hline Wechsler Matrix Reasoning & 141 & $11.0(3-67)$ & $9.0,13.0$ & $11.2(7.0)$ & $25(18 ; 12$ to 25$)$ & .32 \\
\hline Rey-Osterrieth Complex Figure Organization & 325 & $25.0(10-100)$ & $10.0,50.0$ & $41.7(31.6)$ & $184(57 ; 51$ to 62$)$ & $<.0001$ \\
\hline BASC-2 Attention & 316 & $47.0(29-92)$ & $41.0,60.0$ & $49.2(11.9)$ & $85(27 ; 22$ to 32$)$ & $<.0001$ \\
\hline BASC-2 Hyperactivity & 316 & $50.0(31-86)$ & $41.0,55.0$ & $51.2(11.8)$ & $54(17 ; 13$ to 22$)$ & .32 \\
\hline
\end{tabular}

NOTE. The Block Design test was used for patients enrolled onto DFCl protocol 95-01 and the Matrix Reasoning test was used for patients in 00-01. The number and percentage of tested patients who exhibited abnormal neurocognitive or behavioral scores, as defined in the Methods, is shown. The probability that the proportion exhibiting abnormal scores exceeded the expected proportion in the normative population was computed with a one-sided binomial test.

Abbreviations: BASC-2; Behavioral Assessment System for Children-2; DFCl, Dana-Farber Cancer Institute; SD, standard deviation.

standard deviation from the reference population mean. Operationally, this was defined as follows: IQ $\leq 85$; Digit Span, Vocabulary, Block Design, or Matrix Reasoning $\leq 7$; and BASC-2 Attention and Hyperactivity T scores $\geq$ 60. The probability that the proportion exhibiting abnormal scores exceeded the expected proportion in the normative population was computed with a one-sided binomial test.

Univariable and multivariable logistic regression models were constructed for impaired function on each neurocognitive outcome measure. Multivariable logistic regression models were adjusted for age at diagnosis $(<5,5-9.99, \geq 10$ years.), sex (male $v$ female), risk group (standard risk $v$ high risk), race (white $v$ nonwhite), CNS treatment (intrathecal methotrexate $v$ 120-180 Gy $v$ none), type of corticosteroid (prednisone $v$ dexamethasone), socioeconomic status (mother's education, high school or less $v$ some college or more), and genotypes. Although similar, Block Design and Matrix Reasoning assess distinct domains of neurocognitive function. Therefore, they were considered separately in logistic regression modeling. For each polymorphism, the estimated IQ, Vocabulary, and Digit Span scores were also evaluated as continuous variables using linear regression modeling that included the patient characteristics and genotype as described above and were compared univariately using a Wilcoxon rank sum test. All $P$ values were two-sided and considered significant at the .05 level.

The targeted genetic variants were selected a priori on the basis of prior literature and/or hypothesized associations with treatment outcomes. A post hoc Benjamini-Hochberg false discovery rate (FDR) correction for multiple hypothesis testing was made considering the eight neurocognitive outcomes, using the nominal $P$ values obtained from the multivariable logistic regression models. Adjusted $P$ values are denoted as $Q$ values.

\section{RESULTS}

\section{Neurocognitive Outcomes}

Table 3 shows the summary statistics for each neurocognitive measure. Estimated IQ in this cohort did not differ from normative expectations. However, relative to population norms, more patients exhibited impaired Digit Span scores, Rey-Osterrieth Complex Figure organization, and Attention ratings. In addition, 90 (29\%) of the 312 patients who were under 18 years of age at the time of testing were enrolled in special education, a prevalence that is substantially higher than the national rate $(8.4 \% ; P<.001$, one-sided binomial test $) .{ }^{41}$

\section{Genotyping Call Rates}

For each target polymorphism, a specific genotype could be assigned in $44 \%$ to $94 \%$ of patients (median, $83 \%$ ). Three polymorphisms (in MTR, GSTT1, and IL1A) were excluded from further analysis because of genotyping call rates less than $60 \%$. Two polymorphisms (GSTM1 and MAOA rs1799835) were excluded from further analysis because no patients carried the minor allele. Allele frequencies for the remaining 23 targets are shown in Table 2. For all targets, the distribution of genotypes was consistent with Hardy-Weinberg equilibrium, and the minor allele frequencies were similar to published values.

\section{Associations Between Genetic Variants and Neuropsychological Outcomes}

Polymorphisms in two genes (NOS3 and SLCO2A1) were significantly associated with risk for inferior outcomes on five intelligence measures (IQ, Digit Span, Vocabulary, Block Design, and Matrix Reasoning) in multivariable logistic regression modeling (Table 4). The SLCO2A1 polymorphism was also significantly associated with risk for parental reporting of behavioral symptoms of inattention. The COMT polymorphism was marginally associated with risk for parental reporting of both inattention and hyperactivity. Two polymorphisms in HFE were associated with marginally increased risk of hyperactivity behaviors; however, these associations were not significant after FDR adjustment Data Supplement).

The odds of impairment for the measured neurocognitive outcomes were approximately 2 to 5 times higher for patients who carried these target polymorphisms than for those without the target polymorphism in univariable modeling. The odds of IQ impairment were five times higher (adjusted odds ratio $=5.07 ; 95 \% \mathrm{CI}, 1.80$ to 14.28 ; $Q=0.008$ ) for survivors homozygous for the variant NOS3 894T allele in an adjusted model.

Figure 1 illustrates the associations among genetic variants and neurocognitive outcomes measured as continuous variables. Patients with the target NOS3 TT genotype had lower mean scores for estimated IQ (95.8; 95\% CI, 89.9 to $101.7 ; \mathrm{n}=30 v 103.9 ; 95 \% \mathrm{CI}, 102.1$ to $105.8 ; \mathrm{n}=264$; multivariable $P=.011)$ and Vocabulary $(9.5 ; 95 \%$ $\mathrm{CI}, 8.4$ to $10.6 ; \mathrm{n}=30 v 10.9 ; 95 \% \mathrm{CI}, 10.5$ to $11.3 ; \mathrm{n}=268 ; P=.049)$ than patients without this genotype. Patients who carry the variant SLCO2A1 G allele also had lower mean estimated IQ than those who were homozygous for the wild-type A allele (100.4; 95\% CI, 97.5 to $103.2 ; \mathrm{n}=117 v 104.6 ; 95 \% \mathrm{CI}, 102.4$ to $107 ; \mathrm{n}=177 ; P=.014)$, and lower mean Digit Span scores (8.8; 95\% CI, 8.2 to 9.4; $\mathrm{n}=118 v 9.5$; $95 \% \mathrm{CI}, 9.1$ to $10 ; \mathrm{n}=182 ; P=.027)$. Patients with at least one GSTP1 T allele also had lower mean Digit Span scores (8.3; 95\% CI, 7.5 to 9.1; $\mathrm{n}=60$ ) than those who were homozygous for the wild-type $\mathrm{C}$ allele (9.5; 95\% CI, 9.1 to $9.9 ; \mathrm{n}=219 ; P=.006)$ and marginally lower IQ 


\begin{tabular}{|c|c|c|c|c|c|c|}
\hline Neurocognitive Outcome & Gene Symbol (refSNP) & Univariable OR $(95 \% \mathrm{Cl})$ & $P$ & Multivariable OR (95\% Cl) & $P$ & Q \\
\hline \multirow[t]{2}{*}{ Wechsler IO } & NOS3 (rs1799983) & $3.33(1.35$ to 8.32$)$ & .009 & 5.07 (1.80 to 14.28$)$ & .002 & 0.008 \\
\hline & SLCO2A1 (rs7625035) & 2.23 (1.09 to 4.55$)$ & .028 & 2.67 (1.20 to 5.92$)$ & .016 & 0.043 \\
\hline Wechsler Digit Span & SLCO2A1 (rs7625035) & 2.35 (1.41 to 3.91$)$ & $<.001$ & 2.55 (1.48 to 4.40$)$ & $<.001$ & 0.006 \\
\hline Wechsler Vocabulary & NOS3 (rs1799983) & 3.21 (1.39 to 7.41$)$ & .006 & 3.94 (1.54 to 10.09 ) & .004 & 0.011 \\
\hline Wechsler Block Design & SLCO2A1 (rs7625035) & 2.00 (0.92 to 4.34$)$ & .080 & 2.40 (1.05 to 5.48$)$ & .038 & 0.076 \\
\hline Wechsler Matrix Reasoning & NOS3 (rs1799983) & $4.33(1.30$ to 14.42$)$ & .017 & 15.65 (2.80 to 87.66$)$ & .002 & 0.008 \\
\hline \multirow[t]{2}{*}{ Attention (BASC-2) } & COMT (rs4680) & 2.24 (1.12 to 4.48$)$ & .023 & 2.61 (1.26 to 5.45$)$ & .010 & 0.080 \\
\hline & SLCO2A1 (rs7625035) & 1.99 (1.16 to 3.40$)$ & .013 & 2.04 (1.17 to 3.57$)$ & .013 & 0.043 \\
\hline Hyperactivity (BASC-2) & COMT (rs4680) & 2.07 (0.94 to 4.55$)$ & .070 & 2.71 (1.17 to 6.31$)$ & .021 & 0.084 \\
\hline
\end{tabular}

scores (99.9; 95\% CI, 95.7 to $104.2 ; \mathrm{n}=59 v 103.9 ; 95 \% \mathrm{CI}, 101.9$ to $106.0 ; \mathrm{n}=217$; univariable $P=.08)$, although the association with impairment was not significant after FDR adjustment $(P=.029 ; Q=$ 0.23; Data Supplement).

\section{DISCUSSION}

This study demonstrates associations between prevalent genetic polymorphisms and inferior neurocognitive outcomes after treatment for childhood ALL, potentially explaining some of the observed interpatient variability. Our study benefited from a larger sample size than has been included in similar recent studies, ${ }^{17,18,42}$ with 350 pediatric patients treated on two consecutive cooperative group trials. Of note, all of the polymorphisms identified as predictors of inferior neurocognitive outcome have been associated with increased susceptibility to oxidative stress and/or neuroinflammation: endothelial nitric oxide synthase (NOS3), catechol-O-methyltransferase (COMT), hemochromatosis $(H F E)$, glutathione $S$-transferase pi (GSTP1), and the prostaglandin transporter (SLCO2A1).

The NOS3 polymorphism emerged as the variant most strongly related to low IQ among these leukemia survivors. Endothelial nitric oxide production regulates vascular tone and contributes to protec- tion from oxidative damage. This is relevant to patients with leukemia, because we and others have shown in both animal models ${ }^{43}$ and in patients ${ }^{25}$ that treatment with methotrexate leads to an increase in homocysteine within the CNS. Homocysteine may contribute to neurotoxicity by inducing the production of oxygen radicals, leading to oxidative damage of vascular endothelium and neuronal tissue. ${ }^{26,27}$ Homozygosity for the T allele in NOS3 results in decreased enzyme activity, and consequently a diminished capacity for protection against oxidative stress. This polymorphism has been previously linked to decline in IQ after treatment for leukemia in a cohort that included a subset of the patients included in this study $(\mathrm{N}=66){ }^{17}$ Here we expand on this finding, in a larger cohort of survivors treated on the same or subsequent treatment protocol, confirming a significant association between the NOS3 variant and decreased IQ measured cross-sectionally 5 years after completion of treatment.

Mutations in both $H F E^{44}$ and $G S T P 1^{45}$ have also been associated with increased susceptibility to oxidative stress within the CNS. Here we describe a novel association between GSTP1 polymorphisms and lower estimated IQ and Digit Span scores. The $H F E$ variants were marginally associated with increased reports of hyperactive behavior, although the associations were no longer significant after FDR adjustment.

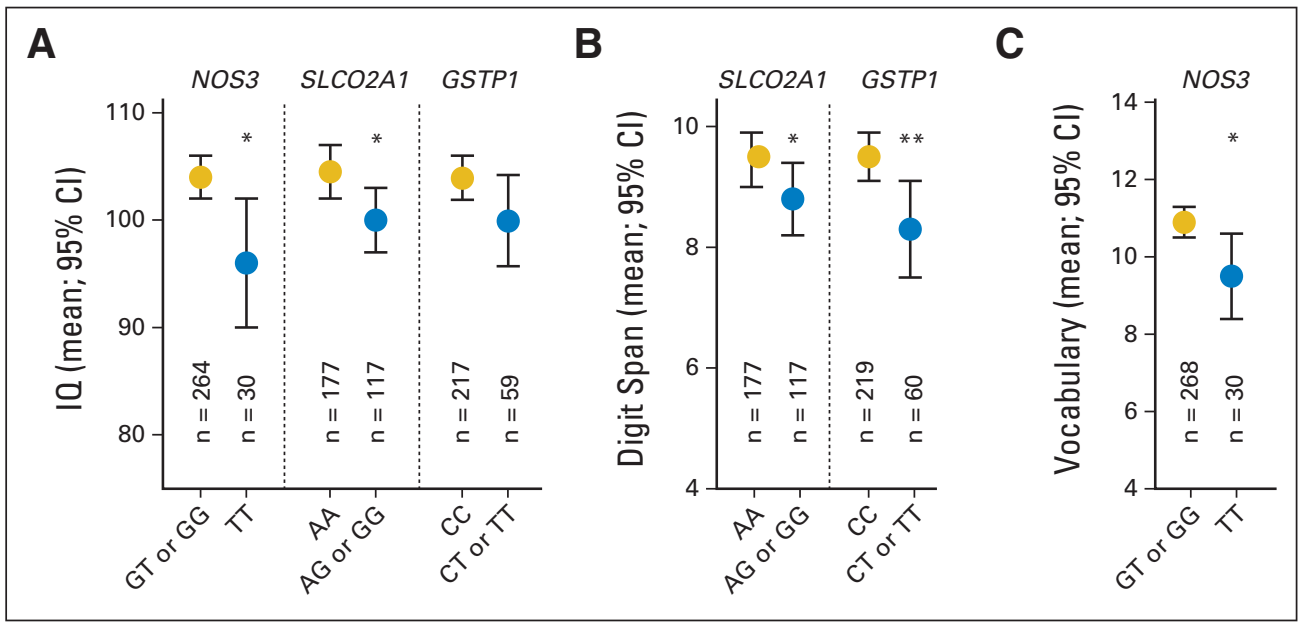

Fig 1. Genetic variants associated with (A) decreased estimated IQ, (B) Wechsler Vocabulary score, and (C) Wechsler Digit Span score. Mean ( \pm SEM) estimated IQ, Digit Span, and Vocabulary scores are shown for patients with (blue circles) or without (gold circles) the target genotype. Mean estimated IQ was lower among patients with the target NOS3 or SLCO2A1 genotype. Mean Digit Span score was lower among patients with the target SLCO2A1 or GSTP1 genotype. Mean Vocabulary score was lower among patients with the target NOS3 genotype. (*) $P<.05$; (**) $P<.01$. 
The function of the COMT gene has primarily been associated with the inactivation of catecholamine neurotransmitters, including dopamine. However, like NOS3, HFE, and GSTP1, it may also relate to susceptibility to oxidative stress. The methyl donor for reactions catalyzed by the gene product, catechol-O-methyltransferase, is $S$-adenosylmethionine, a folate-dependent cofactor that decreases after methotrexate exposure. ${ }^{46}$ The val158Met polymorphism in COMT further decreases enzyme activity, ${ }^{47}$ and the subsequent reduction in $\mathrm{O}$-methylated catechols diminishes protection against oxygen radicals. $^{48,49}$ We observed a marginal association between the val158Met COMT polymorphism and both attention and hyperactivity symptoms among childhood leukemia survivors. These results contrast with a recent report ${ }^{18}$ that did not note such an association. However, this study used different instruments to detect attention problems (Conners Continuous Performance Test and Conners Parent Ratings Scale) than those used in our study (BASC-2 parent report), and had less statistical power to detect this association as a result of a smaller sample size.

In contrast with the genes discussed above, SLCO2A1 has not been as directly associated with oxidative stress. The prostaglandin transporter encoded by this gene facilitates the movement of prostaglandins across the blood brain barrier. Prostaglandins modulate many brain activities by regulating cerebral blood flow, synaptic transmission, neurotrophin production, angiogenesis, and gene expression. Prostaglandins also influence inflammatory processes within the CNS. Because oxygen radicals are produced in the setting of chronic inflammation, it is probable that a functional polymorphism affecting prostaglandin entry into the CNS alters the balance between reactive oxygen species and protective mechanisms.

There were potential limitations to this study. By design, we examined associations with polymorphisms in candidate genes hypothesized to relate to treatment-induced cognitive decline. Unlike an agnostic approach, our study design could not uncover novel polymorphisms linked to cognitive decline, nor point to pathways not previously expected to contribute to toxicity. Second, neurocognitive testing was conducted at a single time point approximately 5 years after treatment, when neurocognitive sequelae are presumably no longer developing. However, because a baseline assessment of cognitive function was not done, associations between genetic variants and inferior cognitive function do not necessarily prove a causal relationship with treatment-induced decline in cognitive function. The con- sistency with the Krajinovic study, however, which included a subsample of our cohort and did measure change over time, supports our findings. Third, there was no nontreated control group to address the possibility that these polymorphisms influence neurocognitive function independently of ALL or chemotherapy. Fifth, pathway and combinatorial analyses were not explored in this study, because statistical power to eliminate false positive associations was limited by the sample size. Finally, there may have been unintended selection bias, as only about half of the patients who were eligible agreed to the neuropsychological testing. It is possible that those patients suspected of having abnormal cognitive function were more likely to consent for testing, falsely increasing the prevalence of cognitive dysfunction in our cohort. However, it seems unlikely that any such selection bias would have systematically altered relationships between cognitive function and genetic variants.

Despite these limitations, the observed associations among genetic polymorphisms and impaired neurocognitive function among leukemia survivors provide additional support for the hypothesis that oxidative stress contributes to treatment-induced cognitive decline among children with ALL. Patients who carry these polymorphisms may be more vulnerable to oxidative stress and thus might benefit from concurrent antioxidant therapy.

\section{AUTHORS' DISCLOSURES OF POTENTIAL CONFLICTS OF INTEREST}

Disclosures provided by the authors are available with this article at www.jco.org.

\section{AUTHOR CONTRIBUTIONS}

Conception and design: Peter D. Cole, Yaron Finkelstein, Traci M. Blonquist, Donna S. Neuberg, Deborah P. Waber

Provision of study materials or patients: Lewis B. Silverman Collection and assembly of data: Peter D. Cole, Veena Vijayanathan, Lewis B. Silverman, Philippe Robaey, Deborah P. Waber Data analysis and interpretation: Peter D. Cole, Yaron Finkelstein, Kristen E. Stevenson, Traci M. Blonquist, Veena Vijayanathan, Lewis B. Silverman, Donna S. Neuberg, Stephen E. Sallan, Deborah P. Waber Manuscript writing: All authors Final approval of manuscript: All authors

\section{REFERENCES}

1. Hudson MM, Link MP, Simone JV: Milestones in the curability of pediatric cancers. J Clin Oncol 32:2391-2397, 2014

2. Duffner $P K$, Armstrong FD, Chen L, et al: Neurocognitive and neuroradiologic central nervous system late effects in children treated on Pediatric Oncology Group (POG) P9605 (standard risk) and P9201 (lesser risk) Acute Lymphoblastic Leukemia Protocols (ACCL0131): A methotrexate consequence? A Report From the Children's Oncology Group. J Pediatr Hematol Oncol 36:8-15, 2014

3. Hodgson KD, Hutchinson AD, Wilson CJ, et al: A meta-analysis of the effects of chemotherapy on cognition in patients with cancer. Cancer Treat Rev 39:297-304, 2013

4. Krull KR, Brinkman TM, Li C, et al: Neurocognitive outcomes decades after treatment for child- hood acute lymphoblastic leukemia: A report from the St. Jude Lifetime Cohort Study. J Clin Oncol 31:4407-4415, 2013

5. Conklin HM, Krull KR, Reddick WE, et al: Cognitive outcomes following contemporary treatment without cranial irradiation for childhood acute Iymphoblastic leukemia. J Natl Cancer Inst 104: 1386-1395, 2012

6. Moyer KH, Willard VW, Gross AM, et al: The impact of attention on social functioning in survivors of pediatric acute lymphoblastic leukemia and brain tumors. Pediatr Blood Cancer 59:12901295, 2012

7. Krull KR, Jain N, Pan $Z$, et al: Executive functions in aging adult survivors of childhood leukemia. J Clin Oncol 28:638s, 2010 (abstr 9011)

8. Ashford J, Schoffstall C, Reddick WE, et al: Attention and working memory abilities in children treated for acute lymphoblastic leukemia. Cancer 116:4638-4645, 2010
9. Buizer Al, de Sonneville LM, Veerman AJ, et al: Effects of chemotherapy on neurocognitive function in children with acute lymphoblastic leukemia: A critical review of the literature. Pediatr Blood Cancer 52:447-454, 2009

10. Lofstad GE, Reinfjell T, Hestad K, et al: Cognitive outcome in children and adolescents treated for acute lymphoblastic leukaemia with chemotherapy only. Acta Paediatr 98:180-186, 2009

11. Peterson $C C$, Johnson $C E$, Ramirez LY, et al: A meta-analysis of the neuropsychological sequelae of chemotherapy-only treatment for pediatric acute lymphoblastic leukemia. Pediatr Blood Cancer 51: 99-104, 2008

12. Mrakotsky CM, Silverman LB, Dahlberg SE, et al: Neurobehavioral side effects of corticosteroids during active treatment for acute lymphoblastic leukemia in children are age-dependent: Report from Dana-Farber Cancer Institute ALL Consortium Protocol 00-01. Pediatr Blood Cancer 57:492-498, 2011 
13. Monje $\mathrm{M}$, Thomason $M E$, Rigolo $\mathrm{L}$, et al: Functional and structural differences in the hippocampus associated with memory deficits in adult survivors of acute lymphoblastic leukemia. Pediatr Blood Cancer 60:293-300, 2013

14. Zeller B, Tamnes CK, Kanellopoulos A, et al: Reduced neuroanatomic volumes in long-term survivors of childhood acute lymphoblastic leukemia. J Clin Oncol 31:2078-2085, 2013

15. Lo Nigro L, Di Cataldo A, Schiliro G: Acute neurotoxicity in children with B-lineage acute lymphoblastic leukemia (B-ALL) treated with intermediate risk protocols. Med Pediatr Oncol 35:449-455, 2000

16. Brouwers P: Commentary: Study of the neurobehavioral consequences of childhood cancer: Entering the genomic era? J Pediatr Psychol 30:79-84, 2005

17. Krajinovic $M$, Robaey $P$, Chiasson $S$, et al: Polymorphisms of genes controlling homocysteine levels and $\mathrm{IQ}$ score following the treatment for childhood ALL. Pharmacogenomics 6:293-302, 2005

18. Krull KR, Bhojwani $D$, Conklin $H M$, et al: Genetic mediators of neurocognitive outcomes in survivors of childhood acute lymphoblastic leukemia. J Clin Oncol 31:2182-2188, 2013

19. Vezmar S, Becker A, Bode $U$, et al: Biochemical and clinical aspects of methotrexate neurotoxic ity. Chemotherapy 49:92-104, 2003

20. Froklage FEAM, Reijneveld JC, Heimans JJ: Central neurotoxicity in cancer chemotherapy: Pharmacogenetic insights. Pharmacogenomics 12:379 395, 2011

21. Quinn CT, Kamen BA: A biochemical perspective of methotrexate neurotoxicity with insight on nonfolate rescue modalities. J Investig Med 44:522 530, 1996

22. Cole PD, Kamen BA: Delayed neurotoxicity associated with therapy for children with acute lymphoblastic leukemia. Ment Retard Dev Disabil Res Rev 12:174-183, 2006

23. Bhojwani D, Sabin ND, Pei D, et al: Methotrexate-induced neurotoxicity and leukoencephalopathy in childhood acute lymphoblastic leukemia. J Clin Oncol 32:949-959, 2014

24. Kishi S, Griener J, Cheng C, et al: Homocysteine, pharmacogenetics, and neurotoxicity in children with leukemia. J Clin Oncol 21:3084-3091, 2003

25. Cole PD, Beckwith KA, Vijayanathan V, et al: Folate homeostasis in cerebrospinal fluid during therapy for acute lymphoblastic leukemia. Pediatr Neurol 40:34-41, 2009

26. Bellamy MF, McDowell IF: Putative mechanisms for vascular damage by homocysteine. J Inherit Metab Dis 20:307-315, 1997

27. Ho Pl, Ashline D, Dhitavat $S$, et al: Folate deprivation induces neurodegeneration: Roles of oxidative stress and increased homocysteine. Neurobiol Dis 14:32-42, 2003

28. Lipton SA, Kim WK, Choi YB, et al: Neurotoxicity associated with dual actions of homocysteine at the N-methyl-D-aspartate receptor. Proc Natl Acad Sci U S A 94:5923-5928, 1997

29. Vijayanathan V, Ali N, Gulinello M, et al: Persistent cognitive deficits, induced by intrathecal methotrexate, are associated with elevated CSF concentrations of excitotoxic glutamate analogs and can be reversed by an NMDA antagonist. Behav Brain Res 225:491-497, 2011

30. Packan DR, Sapolsky RM: Glucocorticoid endangerment of the hippocampus: Tissue, steroid and receptor specificity. Neuroendocrinology 51: 613-618, 1990

31. Hockenberry MJ, Taylor OA, Gundy PM, et al: F2-isoprostanes: A measure of oxidative stress in children receiving treatment for leukemia. Biol Res Nurs 16:303-309, 2013

32. Meyers CA, Albitar M, Estey E: Cognitive impairment, fatigue, and cytokine levels in patients with acute myelogenous leukemia or myelodysplastic syndrome. Cancer 104:788-793, 2005

33. Moghrabi A, Levy DE, Asselin B, et al: Results of the Dana-Farber Cancer Institute ALL Consortium Protocol 95-01 for children with acute lymphoblastic leukemia. Blood 109:896-904, 2007

34. Vrooman LM, Stevenson KE, Supko JG, et al: Postinduction dexamethasone and individualized dosing of Escherichia coli L-asparaginase each improve outcome of children and adolescents with newly diagnosed acute lymphoblastic leukemia: Results from a randomized study_-Dana-Farber Cancer Institute ALL Consortium Protocol 00-01. J Clin Oncol 31:1202-1210, 2013

35. Waber DP, McCabe M, Sebree M, et al: Neuropsychological outcomes of a randomized trial of prednisone versus dexamethasone in acute lymphoblastic leukemia: Findings from Dana-Farber Cancer Institute All Consortium Protocol 00-01. Pediatr Blood Cancer 60:1785-1791, 2013

36. Waber DP, Queally JT, Catania L, et al: Neuropsychological outcomes of standard risk and high risk patients treated for acute lymphoblastic leuke- mia on Dana-Farber ALL Consortium Protocol 95-01 at 5 years post-diagnosis. Pediatr Blood Cancer 58:758-765, 2012

37. Axelrod BN: Validity of the Wechsler abbreviated scale of intelligence and other very short forms of estimating intellectual functioning. Assessment 9:17-23, 2002

38. Wechsler D: Wechsler Intelligence Scale for Children (ed 3). San Antonio, TX, Psychological Corporation, 1991

39. Bernstein JH: Developmental Scoring System for the Rey-Osterrieth Complex Figure. Odessa, FL, Psychological Assessment Resources, 1996

40. Reynolds C, Kamphaus R: BASC: Behavioral Assessment System for Children. Circle Pines, MN, American Guidance Service, 2004

41. Houtenville A, Ruiz T: 2011 Annual Disability Statistics Compendium. Durham, NH, University of New Hampshire, Institute on Disability, 2011

42. Kamdar KY, Krull KR, El-Zein RA, et al: Folate pathway polymorphisms predict deficits in attention and processing speed after childhood leukemia therapy. Pediatric Blood \& Cancer 57:454-460, 2011

43. Li Y, Vijayanathan V, Gulinello M, et al: Intrathecal methotrexate induces focal cognitive deficits and increases cerebrospinal fluid homocysteine. Pharmacol Biochem Behav 95:428-433, 2010

44. Nandar W, Neely EB, Unger E, et al: A mutation in the HFE gene is associated with altered brain iron profiles and increased oxidative stress in mice. Biochim Biochim Biophys Acta 1832:729-741, 2013

45. Strange RC, Spiteri MA, Ramachandran $S$, et al: Glutathione-S-transferase family of enzymes. Mutat Res 482:21-26, 2001

46. Li Y, Vijayanathan V, Gulinello ME, et al: Systemic methotrexate induces spatial memory deficits and depletes cerebrospinal fluid folate in rats. Pharmacol Biochem Behav 94:454-463, 2010

47. Lotta $T$, Vidgren J, Tilgmann $C$, et al: Kinetics of human soluble and membrane-bound catechol O-methyltransferase: A revised mechanism and description of the thermolabile variant of the enzyme. Biochemistry 34:4202-4210, 1995

48. Miller JW, Selhub J, Joseph JA: Oxidative damage caused by free radicals produced during catecholamine autoxidation: Protective effects of O-methylation and melatonin. Free Radic Biol Med 21:241-249, 1996

49. Nappi AJ, Vass E: Hydroxyl radical formation via iron-mediated Fenton chemistry is inhibited by methylated catechols. Biochimica et Biophysica Acta 1425:159-167, 1998

\section{Support}

Supported by St Baldrick's Foundation (P.D.C., Y.F., D.P.W.), National Institutes of Health (NIH) Grant No. IH P30 HD18655 (D.P.W.), NIH Grant No. P01 CA068484 (S.E.S., L.B.S., D.S.N., and K.E.S.), and the Michael J. Garil Fund for Leukemia Research (D.P.W.). The patients described in this report were enrolled at the following Dana-Farber Cancer Institute (DFCI) Acute Lymphoblastic Leukemia Consortium sites: DFCI/Boston Children's Hospital (Boston, MA), Columbia University Medical Center, Morgan Stanley Children's Hospital of New York-Presbyterian (New York, NY), Hospital Sainte Justine (Montreal, QC, Canada), Le Centre Hospitalier de L'Universite Laval (Quebec City, QC, Canada), Maine Children's Cancer Program (Scarborough, ME), McMaster Children's Hospital (Hamilton, ON, Canada), San Jorge Children's Hospital (San Juan, PR), Tulane Hospital for Children (New Orleans, LA), and University of Rochester Medical Center (Rochester, NY). 


\section{AUTHORS' DISCLOSURES OF POTENTIAL CONFLICTS OF INTEREST}

Polymorphisms in Genes Related to Oxidative Stress Are Associated With Inferior Cognitive Function After Therapy for Childhood Acute Lymphoblastic Leukemia

The following represents disclosure information provided by authors of this manuscript. All relationships are considered compensated. Relationships are self-held unless noted. I = Immediate Family Member, Inst = My Institution. Relationships may not relate to the subject matter of this manuscript. For more information about ASCO's conflict of interest policy, please refer to www.asco.org/rwc or jco.ascopubs.org/site/ifc.

\section{Peter D. Cole}

No relationship to disclose

\section{Yaron Finkelstein}

No relationship to disclose

\section{Kristen E. Stevenson}

No relationship to disclose

\section{Traci M. Blonquist}

No relationship to disclose

\section{Veena Vijayanathan}

No relationship to disclose

Lewis B. Silverman

Consulting or Advisory Role: Epizyme, Seattle Genetics Research Funding: Sigma Tau

\section{Donna S. Neuberg}

Stock or Other Ownership: Synta

Patents, Royalties, Other Intellectual Property: Mutations associated with overall survival in myelodsyplastic syndromes (Inst), Mutations associated with response to hypomethylating agents in myelodysplastic syndromes (Inst)

Stephen E. Sallan

No relationship to disclose

Philippe Robaey

No relationship to disclose

Deborah P. Waber

No relationship to disclose 


\section{Appendix}

Table A1. Comparison of Patients Included in Genetic Analyses and Remaining Patients Enrolled Onto 95-01 or 00-01

\begin{tabular}{|c|c|c|c|}
\hline Characteristic & $\begin{array}{l}\text { Neurocognitive and Genetic } \\
\text { Testing Done, No. (\%) }\end{array}$ & Remaining Patients, No. (\%) & $P$ \\
\hline No. & 350 & 633 & \\
\hline \multicolumn{4}{|l|}{ DFCl ALL consortium treatment protocol } \\
\hline $95-01$ & $209(60)$ & $282(45)$ & \multirow[t]{2}{*}{$<.001$} \\
\hline $00-01$ & $141(40)$ & $352(55)$ & \\
\hline Age at initial diagnosis, years; median (range) & $4.2(1-17.99)$ & $5.1(1-17.9)$ & \multirow{4}{*}{$<.001$} \\
\hline$<5$ & $210(60)$ & $313(49)$ & \\
\hline $5-9.99$ & $93(27)$ & $168(27)$ & \\
\hline 10-17.99 & $47(13)$ & $152(24)$ & \\
\hline \multicolumn{4}{|l|}{ Sex } \\
\hline Female & $159(45)$ & $287(45)$ & \multirow[t]{2}{*}{.99} \\
\hline Male & $191(55)$ & $346(55)$ & \\
\hline \multicolumn{4}{|l|}{ DFCl risk group } \\
\hline Standard risk & $225(64)$ & $329(52)$ & \multirow[t]{2}{*}{$<.001$} \\
\hline High risk & $125(36)$ & $304(48)$ & \\
\hline \multicolumn{4}{|l|}{ Race/ethnicity } \\
\hline White & $308(88)$ & $537(85)$ & \multirow[t]{4}{*}{.053} \\
\hline Black or African American & $8(2)$ & $37(6)$ & \\
\hline Asian & $6(2)$ & $7(1)$ & \\
\hline Other/unknown & $28(8)$ & $52(8)$ & \\
\hline Hispanic & $25(7)$ & $73(12)$ & .034 \\
\hline \multicolumn{4}{|l|}{ CNS prophylaxis } \\
\hline Intrathecal chemotherapy & $176(50)$ & 245 (39) & \\
\hline Cranial radiation (12 or $18 \mathrm{~Gy}$ ) & $156(45)$ & $276(44)$ & \\
\hline Did not receive & $18(5)$ & $112(18)$ & \\
\hline \multicolumn{4}{|l|}{ Corticosteroids } \\
\hline Prednisone & $277(79)$ & $489(77)$ & \multirow[t]{3}{*}{.69} \\
\hline Dexamethasone & $73(21)$ & $139(22)$ & \\
\hline Did not receive & $0(0)$ & $5(1)$ & \\
\hline Age at time of neurocognitive testing, years; median (range) & $10(6,25)$ & - & \\
\hline \multicolumn{4}{|l|}{ Mother's education } \\
\hline High school graduate/GED or less & $89(25)$ & - & \\
\hline Some college or more & $251(72)$ & - & \\
\hline Unknown & $10(3)$ & - & \\
\hline Time from diagnosis to neurocognitive testing, years; median (range) & $5(3-9)$ & & \\
\hline $\begin{array}{l}\text { NOTE. The observed differences between these cohorts are a functior } \\
\text { diagnosis. Patients with induction failure, death, or early relapse were th } \\
\text { age) to be more prevalent among the group who did not undergo ne } \\
\text { systematically altered relationships among genetic variants and neurod } \\
\text { Abbreviations: ALL, acute lymphoblastic leukemia; DFCl, Dana-Farber }\end{array}$ & $\begin{array}{l}\text { study design. Neurocognitive } \\
\text { excluded from analysis. Conse } \\
\text { nitive testing. Nevertheless, it } \\
\text { outcomes. Dashes indicate } d \\
\text { Institute; GED, general educa }\end{array}$ & $\begin{array}{l}\text { was conducted among survivor } \\
\text { one would expect high-risk fea } \\
\text { s unlikely that any selection bi } \\
\text { collected. } \\
\text { levelopment. }\end{array}$ & $\begin{array}{l}\text { ars after } \\
\text { eg, older } \\
\text { uld have }\end{array}$ \\
\hline
\end{tabular}

